\title{
OPTIMAL CLOSING OF A MOMENTUM TRADE
}

\author{
ERIK EKSTRÖM, ${ }^{*}$ Uppsala University \\ CARL LINDBERG, ${ }^{* *}$ Chalmers University of Technology
}

\begin{abstract}
There is an extensive academic literature that documents that stocks which have performed well in the past often continue to perform well over some holding period-so-called momentum. We study the optimal timing for an asset sale for an agent with a long position in a momentum trade. The asset price is modelled as a geometric Brownian motion with a drift that initially exceeds the discount rate, but with the opposite relation after an unobservable and exponentially distributed time. The problem of optimal selling of the asset is then formulated as an optimal stopping problem under incomplete information. Based on the observations of the asset, the agent wants to detect the unobservable change point as accurately as possible. Using filtering techniques and stochastic analysis, we reduce the problem to a one-dimensional optimal stopping problem, which we solve explicitly. We also show that the optimal boundary at which the investor should liquidate the trade depends monotonically on the model parameters.
\end{abstract}

Keywords: Optimal stopping; momentum; trading; quickest detection problem for Brownian motion

2010 Mathematics Subject Classification: Primary 91G10

Secondary 60G40

\section{Introduction}

Momentum is the notion that an asset which has performed well in the past will continue to do so for some time. Momentum trades may be the most common asset management strategy, with a large associated literature. The pioneering paper [16] documents that stocks with high recent performance continue to generate significant positive returns over a 3-12 month holding period. This conclusion was further established in [18], and Rouwenhorst [31] found that momentum is also present in international markets. These papers also showed that the momentum effect decays in time, eventually disappearing entirely. Having accepted the existence of momentum as a 'stylized fact' of equity markets, recent academic literature focuses on explaining the phenomenon; a nonexhaustive list is [2], [4], [5], [8], [9], [10], [12], [13], [14], [15], [17], [19], [23], [24], [25], [27], and [28].

Clearly, one way to benefit from a detected momentum opportunity is to buy the stock and then liquidate it at some pre-determined future time. An obvious risk with such a strategy, however, is that the momentum effect disappears much earlier or much later than the pre-determined liquidation time.

In this paper we analyze how to choose optimal liquidation strategies for momentum trades. More precisely, we seek to maximize the expected profit from a momentum trade. The expected return of a stock is assumed to be a constant larger than the discount rate up until some random,

Received 3 November 2011; revision received 31 August 2012.

* Postal address: Uppsala University, Box 480, SE-75106 Uppsala, Sweden. Email address: ekstrom@math.uu.se

** Current address: Second Swedish National Pension Fund - AP2, SE 40424 Göteborg, Sweden. 
and unobservable, time $\theta$, at which it drops to a constant smaller than the discount rate. The assumption that the momentum trend eventually disappears is in accordance with the consensus in the financial literature; see, e.g. [16], [18], and [31] (for an alternative model for momentum, see [36]). Based on this model for the expected return, we seek the optimal liquidation time for an investor with a long position in a momentum trade. Clearly, the investor wants to hold the position as long as the momentum is present, thereby taking advantage of the drift exceeding the discount rate. On the other hand, when the momentum disappears, the investor would like to exit the position to avoid an investment in an asset with an unfavourable drift. However, the time point $\theta$ at which the momentum disappears is not directly observable, so to exit the position exactly at $\theta$ is not possible in general. Instead, the agent has to make a guess based merely on his/her observations of the asset price fluctuations.

We formulate the optimal liquidation problem as an optimal stopping problem under incomplete information. Applying the filtering techniques used in the quickest detection problem for a Brownian motion, see [32], we arrive at an optimal stopping problem under complete information. In addition to the underlying asset, this problem also involves the Markov process given by the conditional probability that $\theta$ has occurred given the asset price observations up to the current time. Next, using a Girsanov change of measure and stochastic analysis, we reduce the number of spatial dimensions and express the optimal liquidation problem in terms of an auxiliary one-dimensional optimal stopping problem with an affine payoff function for a diffusion. An explicit solution to this auxiliary problem is found by solving the related free boundary problem involving an ordinary differential equation. We show that, for the auxiliary problem, the optimal stopping boundary is a constant barrier which depends monotonically on each of the model parameters. Accordingly, the optimal liquidation strategy for the momentum trade is to sell the asset the first time $t$ the conditional probability that $\theta \leq t$ exceeds a certain level. We also analyze the dependence of the solution on the different model parameters. This is important, both from a mathematical and from a practical perspective, in order to obtain an understanding of the model sensitivity to changes in the parameter input and to possible mis-specifications. In particular, we show that the optimal liquidation level is decreasing in the intensity at which the momentum opportunity disappears, and it is also decreasing with respect to the volatility of the underlying asset.

The problem under study in the present paper was also addressed in [1]. The authors of [1] showed that the optimal liquidation time, for most parameter choices, coincides with the first hitting time of some level for the conditional probability that $\theta$ has occurred. However, no characterization of this level or means of calculating it were provided. The same problem is also discussed in [34]. The authors of [34] formulated the liquidation problem as an optimal stopping problem for a two-dimensional Markov process. In view of the results of these references, the main mathematical contribution of the current paper is to show that the two-dimensional optimal stopping problem can be reduced to a one-dimensional problem using the Girsanov theorem, together with a detailed analysis of the resulting one-dimensional problem, thereby providing a full solution of the liquidation problem. For related studies where optimal stopping theory is applied to optimal stock selling problems, see, e.g. [6], [7], [11], [33], and [35].

We define the asset price model in Section 2, and the optimal liquidation problem is setup. Moreover, filtering techniques and the Girsanov theorem are used to formulate the problem in terms of a one-dimensional auxiliary optimal stopping problem. In Section 3, the auxiliary optimal stopping problem is studied, and the optimal stopping time is determined. In Section 4 we study the dependency of the solution on the different model parameters. Finally, in Section 5 we study a similar case but with an investor who has a short position in a momentum trade. 


\section{The model}

We take as given a complete probability space $(\Omega, \mathcal{F}, \mathbb{P})$. On this probability space, let $\theta$ be a random variable with distribution $\mathbb{P}(\theta=0)=\pi$ and $\mathbb{P}(\theta \geq t \mid \theta>0)=\mathrm{e}^{-\lambda t}$. We assume that the intensity $\lambda$ is positive and that $\pi$ belongs to $[0,1)$.

Next, let $W$ be a Brownian motion which is independent of $\theta$. The asset price process $X$ is modelled by a geometric Brownian motion with a drift that drops from $\mu_{2}$ to $\mu_{1}$ at time $\theta$. More precisely,

$$
\mathrm{d} X_{t}=\mu(t) X_{t} \mathrm{~d} t+\sigma X_{t} \mathrm{~d} W_{t}
$$

and $X_{0}=x>0$, where

$$
\mu(t)=\mu_{2}-\left(\mu_{2}-\mu_{1}\right) \mathbf{1}_{\{t \geq \theta\}}
$$

and $\mathbf{1}_{\{.\}}$is the indicator function. Here $\mu_{1}, \mu_{2}$, and $\sigma>0$ are constants with $\mu_{1}<\mu_{2}$. Denote by $\mathcal{F}^{X}=\left(\mathcal{F}_{t}^{X}\right)_{t \in[0, \infty)}$ the filtration generated by $X$, and note that $\mathcal{F}^{X}$ is strictly contained in the filtration generated by $X$ and $\theta$.

Now, let $\mathcal{T}$ be the set of (possibly infinite) $\mathcal{F}^{X}$-stopping times. We consider the optimal stopping problem

$$
V=\sup _{\tau \in \mathcal{T}} \mathbb{E} \mathrm{e}^{-r \tau} X_{\tau}
$$

where the discount rate $r \geq 0$ is a given constant. Here (and in all other similar situations below) we use the convention that $\mathrm{e}^{-r \tau} X_{\tau}=0$ on the event $\{\tau=\infty\}$.

Note that if $r<\mu_{1}<\mu_{2}$ then $\mathrm{e}^{-r t} X_{t}$ is an $\mathcal{F}^{X}$-submartingale, and $V=\infty$ (choose deterministic stopping times $\tau=t$, and let $t \rightarrow \infty)$. If $r=\mu_{1}<\mu_{2}$ then $\mathrm{e}^{-r t} X_{t}$ is an $\mathcal{F}^{X}$-submartingale and again the supremum is attained along a sequence of deterministic times (the value $V$ is then infinite if $\mu_{2} \geq r+\lambda$, and $V=\pi x+(1-\pi) x \lambda /\left(\lambda+r-\mu_{2}\right)$ if $\left.\mu_{2}<r+\lambda\right)$. Similarly, if $\mu_{1}<\mu_{2} \leq r$ then $\mathrm{e}^{-r t} X_{t}$ is a supermartingale, $V=x$, and the supremum is attained for $\tau=0$. Therefore, throughout the remainder of this article, we assume that $\mu_{1}<r<\mu_{2}$.

Remark. We emphasize that the supremum in (1) is with respect to $\mathcal{F}^{X}$-stopping times. This models a situation in which the agent cannot directly observe the change point $\theta$. Instead, he/she merely observes the asset price trajectory, and based on these observations he/she tries to determine an optimal liquidation strategy.

On the other hand, an agent with complete information about the change point would choose to liquidate exactly at $\theta$. The expected payoff for such an agent would then be

$$
\begin{aligned}
\mathbb{E}^{-r \theta} X_{\theta} & =\pi x+(1-\pi) x \int_{0}^{\infty} \mathrm{e}^{\left(\mu_{2}-r\right) t} \lambda \mathrm{e}^{-\lambda t} \mathrm{~d} t \\
& = \begin{cases}\pi x+\frac{(1-\pi) x \lambda}{\lambda+r-\mu_{2}} & \text { if } \mu_{2}<r+\lambda, \\
\infty & \text { if } \mu_{2} \geq r+\lambda\end{cases}
\end{aligned}
$$

Clearly, this value is an upper bound for $V$.

Remark. Another possible interpretation of the liquidation problem described above would be that $X$ models the price of an asset bubble. As long as the bubble lives, the agent clearly wants to keep the asset, but once the bubble bursts, it is optimal to sell it. 
Remark. The optimal liquidation problem (1) is also studied in [1]. The authors of [1] showed that the optimal liquidation time is the first passage time over some unknown level for the a posteriori probability process $\Pi$ defined below. However, no characterization of the optimal liquidation level is obtained.

In [34] a similar problem is studied, but with the difference that the asset price is modelled by a linear Brownian motion instead of a geometric Brownian motion. The authors also briefly discussed the case of a geometric Brownian motion as in (1), and derived a representation in terms of a two-dimensional optimal stopping problem. One key observation of the present paper is that, in fact, problem (1) can be reduced to a one-dimensional optimal stopping problem, and, therefore, also be solved explicitly.

We define the a posteriori probability process $\Pi$ by

$$
\Pi_{t}=\mathbb{P}\left(\theta \leq t \mid \mathcal{F}_{t}^{X}\right) .
$$

Note that $\Pi_{0}=\pi$. It is well known from filtering theory, see [26, Chapter 9], that $\Pi$ satisfies

$$
\mathrm{d} \Pi_{t}=\lambda\left(1-\Pi_{t}\right) \mathrm{d} t-\omega \Pi_{t}\left(1-\Pi_{t}\right) \mathrm{d} \bar{W}_{t},
$$

where $\omega=\left(\mu_{2}-\mu_{1}\right) / \sigma$ and $\bar{W}_{t}$ is a $\mathbb{P}$-Brownian motion with respect to $\mathcal{F}_{t}^{X}$ given by

$$
\begin{aligned}
\mathrm{d} \bar{W}_{t} & =\frac{\mathrm{d} X_{t}}{\sigma X_{t}}-\frac{1}{\sigma}\left(\Pi_{t} \mu_{1}+\left(1-\Pi_{t}\right) \mu_{2}\right) \mathrm{d} t \\
& =\frac{1}{\sigma}\left(\mu(t)-\Pi_{t} \mu_{1}-\left(1-\Pi_{t}\right) \mu_{2}\right) \mathrm{d} t+\mathrm{d} W_{t} .
\end{aligned}
$$

Moreover, in terms of $\bar{W}$ we have

$$
\mathrm{d} X_{t}=\left(\Pi_{t} \mu_{1}+\left(1-\Pi_{t}\right) \mu_{2}\right) X_{t} \mathrm{~d} t+\sigma X_{t} \mathrm{~d} \bar{W}_{t},
$$

and the filtration generated by $\bar{W}$ coincides with $\mathcal{F}^{X}$. Furthermore, if

$$
\Phi_{t}:=\frac{\Pi_{t}}{1-\Pi_{t}}
$$

then $\Phi_{0}=\phi:=\pi /(1-\pi)$ and Itô's formula gives

$$
\mathrm{d} \Phi_{t}=\left(\frac{\lambda}{1-\Pi_{t}}+\omega^{2} \Pi_{t} \Phi_{t}\right) \mathrm{d} t-\omega \Phi_{t} \mathrm{~d} \bar{W}_{t} .
$$

Next, we note that

$$
M_{t}:=\exp \left\{-\frac{1}{2} \int_{0}^{t}\left(\sigma+\omega \Pi_{s}\right)^{2} \mathrm{~d} s+\int_{0}^{t}\left(\sigma+\omega \Pi_{s}\right) \mathrm{d} \bar{W}_{s}\right\}
$$

is an $\mathcal{F}^{X}$-martingale, and apply a generalized version of Girsanov's theorem (Corollary 3.5.2 of [21]) to find the unique probability measure $\mathbb{Q}$ such that

$$
\mathbb{Q}(A)=\mathbb{E}\left[\mathbf{1}_{A} M_{t}\right]
$$

for $A \in \mathcal{F}_{t}^{X}, 0 \leq t<\infty$. Under $\mathbb{Q}$, the new process $Z$ defined by

$$
\mathrm{d} Z_{t}=-\left(\sigma+\omega \Pi_{t}\right) \mathrm{d} t+\mathrm{d} \bar{W}
$$

is a $\left\{\mathbb{Q}, \mathcal{F}^{X}\right\}$-Brownian motion. Furthermore,

$$
\mathrm{d} \Phi_{t}=\left(\lambda+(\lambda-\omega \sigma) \Phi_{t}\right) \mathrm{d} t-\omega \Phi_{t} \mathrm{~d} Z_{t} .
$$


Our first result shows that the Radon-Nikodym derivative $Y$ can be expressed conveniently in terms of $\Phi$ and $X$. This important observation is motivated by the results of Klein, who showed a similar result in [22] in a setting with incomplete information about a constant (but random) drift.

Proposition 1. Let $\tau$ be an $\mathcal{F}^{X}$-stopping time. Then

$$
\mathbb{E} \mathrm{e}^{-r \tau} X_{\tau}=\frac{x}{1+\phi} \mathbb{E}^{\mathbb{Q}} \mathrm{e}^{\left(\mu_{2}-\lambda-r\right) \tau}\left(1+\Phi_{\tau}\right)
$$

Proof. Let $\eta_{t}=1 / M_{t}$. Then

$$
\begin{aligned}
\eta_{t} & =\exp \left\{\frac{1}{2} \int_{0}^{t}\left(\sigma+\omega \Pi_{t}\right)^{2} \mathrm{~d} t-\int_{0}^{t}\left(\sigma+\omega \Pi_{t}\right) \mathrm{d} \bar{W}_{t}\right\} \\
& =\exp \left\{-\frac{1}{2} \int_{0}^{t}\left(\sigma+\omega \Pi_{t}\right)^{2} \mathrm{~d} t-\int_{0}^{t}\left(\sigma+\omega \Pi_{t}\right) \mathrm{d} Z_{t}\right\}
\end{aligned}
$$

and

$$
\frac{\mathrm{d} \eta_{t}}{\eta_{t}}=-\left(\sigma+\omega \Pi_{t}\right) \mathrm{d} Z_{t}
$$

Moreover,

$$
\mathbb{E} \mathrm{e}^{-r \tau} X_{\tau}=\mathbb{E}^{\mathbb{Q}} \eta_{\tau} \mathrm{e}^{-r \tau} X_{\tau}
$$

Now, consider the process

$$
Y_{t}=\frac{\left(1+\Phi_{t}\right) \mathrm{e}^{\left(\mu_{2}-\lambda\right) t} x}{(1+\phi) X_{t}} .
$$

Using Itô's formula, it is straightforward to check that

$$
\frac{\mathrm{d} Y_{t}}{Y_{t}}=-\left(\sigma+\omega \Pi_{t}\right) \mathrm{d} Z_{t}=\frac{\mathrm{d} \eta_{t}}{\eta_{t}} .
$$

Since $Y_{0}=1=\eta_{0}$, it follows that $\eta_{t}=Y_{t}$ for all $t$ almost surely. In view of (2), the result follows.

It follows from Proposition 1 that

$$
V=\frac{x}{1+\phi} \sup _{\tau \in \mathcal{T}} \mathbb{E}^{\mathbb{Q}} \mathrm{e}^{\left(\mu_{2}-\lambda-r\right) \tau}\left(1+\Phi_{\tau}\right) .
$$

In the next section we study the optimal stopping problem in the above form. The implications for problem (1) are summarized in Corollary 1 below.

\section{The auxiliary optimal stopping problem}

In this section we study the optimal stopping problem in (3). Thus, on some probability space, let $\Phi_{t}$ be a diffusion process satisfying

$$
\mathrm{d} \Phi_{t}=\left(\lambda+(\lambda-\omega \sigma) \Phi_{t}\right) \mathrm{d} t-\omega \Phi_{t} \mathrm{~d} Z_{t},
$$

where $Z$ is a standard Brownian motion. Define

$$
F(\phi)=\sup _{\tau} \mathbb{E}_{\phi} \mathrm{e}^{\left(\mu_{2}-\lambda-r\right) \tau}\left(1+\Phi_{\tau}\right),
$$

where the index indicates that $\Phi_{0}=\phi$, and the supremum is taken over random variables that are stopping times with respect to the filtration generated by $Z$. Note that $V$ defined in (1) satisfies $V=x F(\phi) /(1+\phi)$; cf. (3). 
The quantity $F$ is infinite if $\mu_{2} \geq \lambda+r$ since $\Phi$ will reach any positive level with probability 1 (choose $\tau=\inf \left\{t: \Phi_{t} \geq b\right\}$ and let $b \rightarrow \infty$ ).

We therefore assume throughout this section that $\mu_{2}<\lambda+r$. It then follows from an application of Itô's formula that the drift of the process $\mathrm{e}^{\left(\mu_{2}-\lambda-r\right) t}\left(1+\Phi_{t}\right)$ is positive if $\Phi_{t}<\left(\mu_{2}-r\right) /\left(r-\mu_{1}\right)$ and negative if $\Phi_{t}>\left(\mu_{2}-r\right) /\left(r-\mu_{1}\right)$. This suggests that the optimal stopping time in (4) is of the form

$$
\tau_{B}:=\inf \left\{t \geq 0: \Phi_{t} \geq B\right\}
$$

for some level $B>\left(\mu_{2}-r\right) /\left(r-\mu_{1}\right)$; cf. [37]. Moreover, optimal stopping theory (see [30]) suggests that the pair $(F, B)$ satisfies the free boundary problem

$$
\begin{gathered}
\mathcal{L} F+\left(\mu_{2}-\lambda-r\right) F=0, \quad 0<\phi<B, \\
F(\phi)=1+\phi, \quad \phi \geq B ; \quad F^{\prime}(B)=1, \quad F(0+)<\infty,
\end{gathered}
$$

where

$$
\mathcal{L} F=\frac{\omega^{2} \phi^{2}}{2} F^{\prime \prime}+(\lambda+(\lambda-\omega \sigma) \phi) F^{\prime} .
$$

The ordinary differential equation in (5) has the general solution

$$
F(\phi)=C \psi(\phi)+D \varphi(\phi),
$$

where

$$
\psi(\phi)=\int_{0}^{\infty} \mathrm{e}^{-a t} t^{(b+\gamma-3) / 2}(1+\phi t)^{(\gamma-b+1) / 2} \mathrm{~d} t
$$

and

$$
\varphi(\phi)=\int_{0}^{1 / \phi} \mathrm{e}^{a t} t^{(b+\gamma-3) / 2}(1-\phi t)^{(\gamma-b+1) / 2} \mathrm{~d} t
$$

with

$\gamma=\sqrt{(b-1)^{2}+4 c}, \quad a=\frac{2 \lambda}{\omega^{2}}, \quad b=\frac{2}{\omega}\left(\frac{\lambda}{\omega}-\sigma\right), \quad$ and $\quad c=\frac{2\left(\lambda+r-\mu_{2}\right)}{\omega^{2}}>0$.

(The functions $\varphi$ and $\psi$ can alternatively be expressed in terms of the confluent hypergeometric functions of the first and second kind, respectively.)

Lemma 1. The function $\psi$ is increasing and convex on $(0, \infty)$. The function $\varphi$ is decreasing and satisfies $\varphi(0+)=\infty$.

Proof. We have

$$
\psi^{\prime}(\phi)=\frac{\gamma-b+1}{2} \int_{0}^{\infty} \mathrm{e}^{-a t} t^{(b+\gamma-1) / 2}(1+\phi t)^{(\gamma-b-1) / 2} \mathrm{~d} t,
$$

which is positive since $\gamma=\sqrt{(b-1)^{2}+4 c}>b-1$. Hence, $\psi$ is increasing. Moreover,

$$
\frac{2 \psi^{\prime \prime}(\phi)}{\gamma-b+1}=\frac{\gamma-b-1}{2} \int_{0}^{\infty} \mathrm{e}^{-a t} t^{(b+\gamma+1) / 2}(1+\phi t)^{(\gamma-b-3) / 2} \mathrm{~d} t .
$$

Now note that $\mu_{1}<r$ implies that $b<c$, which in turn implies that $\gamma>b+1$. Consequently, $\psi$ is convex.

Similarly,

$$
\varphi^{\prime}(\phi)=\frac{b-1-\gamma}{2} \int_{0}^{1 / \phi} \mathrm{e}^{a t} t^{(b+\gamma-1) / 2}(1-\phi t)^{(\gamma-b-1) / 2} \mathrm{~d} t<0,
$$


so $\varphi$ is decreasing. Moreover, for small $\phi$, we have

$$
\begin{aligned}
\varphi(\phi) & =\int_{0}^{1 / \phi} \mathrm{e}^{a t} t^{(b+\gamma-3) / 2}(1-\phi t)^{(\gamma-b+1) / 2} \mathrm{~d} t \\
& \geq(1-\sqrt{\phi})^{(\gamma-b+1) / 2} \int_{0}^{1 / \sqrt{\phi}} t^{(b+\gamma-3) / 2} \mathrm{~d} t \\
& \geq \varepsilon \phi^{(1-\gamma-b) / 4},
\end{aligned}
$$

where $\varepsilon$ is some positive constant. Since $\gamma>1-b$, it follows that $\varphi(0+)=\infty$.

Remark. The function $\psi$ is the unique (up to multiplication with a positive constant), positive, and increasing solution of $\mathcal{L} f+\left(\mu_{2}-\lambda-r\right) f=0$; cf. pages 18-19 of [3]. Similarly, $\varphi$ is the unique, positive, and decreasing solution.

Note that the condition $F(0+)<\infty$ implies that we must have $D=0$. The two boundary conditions at $B$ in (5) then imply that the two remaining unknowns $B$ and $C$ satisfy

$$
C \psi(B)=1+B, \quad C \psi^{\prime}(B)=1 \text {. }
$$

Using (8), we find that

$$
\begin{gathered}
C \int_{0}^{\infty} \mathrm{e}^{-a t} t^{(b+\gamma-3) / 2}(1+B t)^{(\gamma-b+1) / 2} \mathrm{~d} t=1+B, \\
C \frac{\gamma-b+1}{2} \int_{0}^{\infty} \mathrm{e}^{-a t} t^{(b+\gamma-1) / 2}(1+B t)^{(\gamma-b-1) / 2} \mathrm{~d} t=1,
\end{gathered}
$$

and solving for $B$ gives the free boundary equation

$$
\begin{aligned}
2 \int_{0}^{\infty} & \mathrm{e}^{-a t} t^{(b+\gamma-3) / 2}(1+B t)^{(\gamma-b+1) / 2} \mathrm{~d} t \\
& =(\gamma-b+1)(1+B) \int_{0}^{\infty} \mathrm{e}^{-a t} t^{(b+\gamma-1) / 2}(1+B t)^{(\gamma-b-1) / 2} \mathrm{~d} t .
\end{aligned}
$$

Lemma 2. There exists a unique positive solution B to the free boundary equation (9).

Proof. Define the function $g:(0, \infty) \rightarrow \mathbb{R}$ by

$$
\begin{aligned}
g(x):= & 2 \int_{0}^{\infty} \mathrm{e}^{-a t} t^{(b+\gamma-3) / 2}(1+x t)^{(\gamma-b+1) / 2} \mathrm{~d} t \\
& -(\gamma-b+1)(1+x) \int_{0}^{\infty} \mathrm{e}^{-a t} t^{(b+\gamma-1) / 2}(1+x t)^{(\gamma-b-1) / 2} \mathrm{~d} t .
\end{aligned}
$$

Then

$$
\begin{aligned}
g(0+) & =2 \int_{0}^{\infty} \mathrm{e}^{-a t} t^{(b+\gamma-3) / 2} \mathrm{~d} t-(\gamma-b+1) \int_{0}^{\infty} \mathrm{e}^{-a t} t^{(b+\gamma-1) / 2} \mathrm{~d} t \\
& =\left(\frac{4 a}{b+\gamma-1}-(\gamma-b+1)\right) \int_{0}^{\infty} \mathrm{e}^{-a t} t^{(b+\gamma-1) / 2} \mathrm{~d} t
\end{aligned}
$$

where the last equality follows by integration by parts. Since $4 a>4 c=(\gamma-b+1)(\gamma+b-1)$, it follows that $g(0+)>0$. Moreover,

$$
g(x) \sim-x^{(\gamma-b-1) / 2}(\gamma-b-1) \int_{0}^{\infty} \mathrm{e}^{-a t} t^{\gamma-1} \mathrm{~d} t
$$

for large $x$. Since $\gamma>b+1$, it follows that $g(x)$ is negative for large $x$. Existence of a solution now follows from the continuity of $g$. 

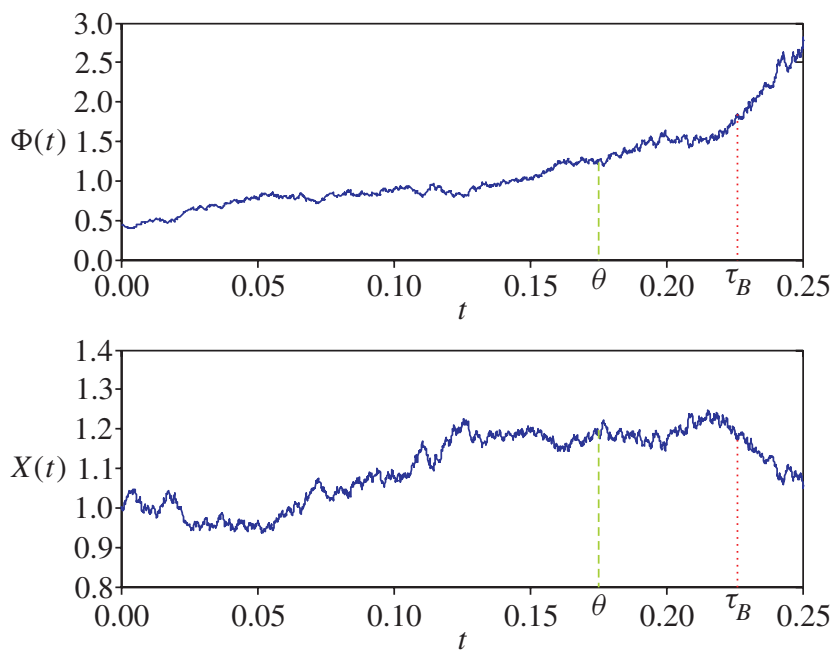

Figure 1: Top: a simulated path of the process $\Phi_{t}$ for $\mu_{1}=-0.2, \mu_{2}=0.2, \sigma=0.4, r=0.05$, and $\lambda=3$. These parameters yield $B=1.84$ using (9). Bottom: the stock price $X_{t}$ for the same simulation as above. The change point $\theta$ and the optimal stopping time $\tau_{B}$ are marked with dashed and dotted lines, respectively.

Uniqueness of solutions follows from the fact that

$$
g^{\prime}(x)=-(\gamma-b+1)(1+x) \int_{0}^{\infty} \mathrm{e}^{-a t} t^{(b+\gamma+1) / 2}(1+x t)^{(\gamma-b-3) / 2} \mathrm{~d} t<0
$$

for all $x \in(0, \infty)$.

We next show that the first hitting time of the level $B$ is an optimal stopping time. For a graphic illustration of the optimal stopping time, see Figure 1.

Theorem 1. The function $F$ defined in (7) satisfies

$$
F(\phi)= \begin{cases}\frac{1+B}{\psi(B)} \psi(\phi), & \phi<B, \\ 1+\phi, & \phi \geq B,\end{cases}
$$

where $B$ is the unique solution to the free boundary equation (9). Moreover, we have $B \geq$ $\left(\mu_{2}-r\right) /\left(r-\mu_{1}\right)$, and the stopping time $\tau_{B}:=\inf \left\{t \geq 0: \Phi_{t} \geq B\right\}$ is optimal in (4).

Proof. Let $B$ be the unique solution to (9), and define $G$ by

$$
G(\phi):= \begin{cases}\frac{1+B}{\psi(B)} \psi(\phi), & \phi<B \\ 1+\phi, & \phi \geq B .\end{cases}
$$

Then $G(\phi) \geq 1+\phi$, and $G(\phi)>1+\phi$ if and only if $\phi<B$. By (a generalized version of) Itô's formula, the process $Y_{t}=\mathrm{e}^{\left(\mu_{2}-\lambda-r\right) t} G\left(\Phi_{t}\right)$ satisfies

$$
\mathrm{d} Y_{t}=\mathrm{e}^{\left(\mu_{2}-\lambda-r\right) t}\left(\mu_{2}-r+\left(\mu_{1}-r\right) \Phi_{t}\right) \mathbf{1}_{\left\{\Phi_{t}>B\right\}} \mathrm{d} t-\mathrm{e}^{\left(\mu_{2}-\lambda-r\right) t} \omega \Phi_{t} G^{\prime}\left(\Phi_{t}\right) \mathrm{d} Z_{t} .
$$

Now assume that the solution $B$ to the free boundary equation (9) satisfies $B \geq\left(\mu_{2}-r\right) /\left(r-\mu_{1}\right)$ (this is indeed verified below). Then the drift of $Y$ is negative, and $Y$ is a supermartingale. Moreover, $Y_{t \wedge \tau_{B}}$ is a martingale with a last element. 
Now, let $\tau$ be a stopping time. An application of the optional sampling theorem (see Problem 1.3.16 and Theorem 1.3.22 of [21]) yields

$$
\mathbb{E}_{\phi} \mathrm{e}^{\left(\mu_{2}-\lambda-r\right) \tau}\left(1+\Phi_{\tau}\right) \leq \mathbb{E}_{\phi} Y_{\tau} \leq \mathbb{E}_{\phi} Y_{0}=G(\phi)
$$

Consequently, $F(\phi) \leq G(\phi)$.

Conversely, for $\tau=\tau_{B}$, the inequalities in (11) are in fact equalities. Therefore,

$$
F(\phi)=\sup _{\tau} \mathbb{E}_{y}^{\mathbb{Q}} Y_{\tau} \geq \mathbb{E}_{y}^{\mathbb{Q}} Y_{\tau_{B}}=G(\phi) .
$$

It follows that $F(\phi)=G(\phi)$.

It remains to show that $B \geq\left(\mu_{2}-r\right) /\left(r-\mu_{1}\right)=: d$. To this end, first recall that, by general optimal stopping theory, see, e.g. [20, Theorem D.12], it is optimal to stop the first time $\Phi$ enters the stopping region $\{\phi \in(0, \infty): F(\phi)=1+\phi\}$. Moreover, points where $\left(\mathcal{L}+\mu_{2}-\lambda-r\right)(1+\phi)=\mu_{2}-r+\left(\mu_{1}-r\right) \phi$ is positive belong to the continuation region $\{y \in(0, \infty): F(\phi)>1+\phi\}$ (this follows by a local argument; see, e.g. the remark after Theorem 10.1.12 of [29]). It follows that $F$ satisfies

$$
F(\phi)=\sup _{\left\{\tau: \Phi_{\tau} \geq d\right\}} \mathbb{E}_{\phi} \mathrm{e}^{\left(\mu_{2}-\lambda-r\right) \tau}\left(1+\Phi_{\tau}\right),
$$

i.e. it suffices to take the supremum over stopping times $\tau$ such that $\Phi_{\tau} \geq d$. Define the function

$$
G^{d}(\phi):= \begin{cases}\frac{1+d}{\psi(d)} \psi(\phi), & \phi<d, \\ 1+\phi, & \phi \geq d .\end{cases}
$$

Now, assume that the unique solution $B$ to the free boundary equation satisfies $B<d$. In that case, the function $g$ in $(10)$ satisfies $g(d)<0$. Consequently, the left derivative of $G^{d}$ at the point $d$ is larger than the right derivative. In particular, $G^{d}(\phi)<1+\phi$ for some $\phi<d$. It also follows (again, by a generalized version of Itô's formula) that the process e ${ }^{\left(\mu_{2}-\lambda-r\right) t} G^{d}\left(\Phi_{t}\right)$ is a supermartingale, and $\mathrm{e}^{\left(\mu_{2}-\lambda-r\right)\left(t \wedge \tau_{d}\right)} G^{d}\left(\Phi_{t \wedge \tau_{d}}\right)$ is a martingale. For stopping times $\tau$ satisfying $\Phi_{\tau} \geq d$, we therefore obtain

$$
\mathbb{E}_{\phi} \mathrm{e}^{\left(\mu_{2}-\lambda-r\right) \tau}\left(1+\Phi_{\tau}\right)=\mathbb{E}_{\phi} \mathrm{e}^{\left(\mu_{2}-\lambda-r\right) \tau} G^{D}\left(\Phi_{\tau}\right) \leq G^{d}(\phi) .
$$

In view of (12), the inequality $F(\phi) \leq G^{d}(\phi)$ follows. However, this is a contradiction since there exist points with $G^{d}(\phi)<1+\phi$, whereas $F(\phi) \geq 1+\phi$ everywhere. This completes the proof.

Remark. A more direct approach to prove that $B \geq\left(\mu_{2}-r\right) /\left(r-\mu_{1}\right)$ would be to show that $g\left(\left(\mu_{2}-r\right) /\left(r-\mu_{1}\right)\right) \geq 0$, where $g$ is defined in (10). However, we did not find a short argument for this.

The following result is a consequence of Proposition 1 and Theorem 1.

Corollary 1. Let $B$ be the unique positive solution to (9), and let $\phi=\pi /(1-\pi)$. Then the value $V$ defined in (1) satisfies

$$
V= \begin{cases}\frac{(1+B) \psi(\phi)}{(1+\phi) \psi(B)} x, & \phi<B, \\ x, & \phi \geq B .\end{cases}
$$


Moreover, the stopping time

$$
\tau^{*}=\inf \left\{t \geq 0: \Pi_{t} \geq \frac{B}{B+1}\right\}
$$

is optimal in (1).

\section{Parameter dependencies}

In this section we study the dependence of the optimal liquidation strategy $B$ and the value $V$ on the different parameters of the model. Figure 2 illustrates numerically the dependence of $B$ on $\sigma$ and $\lambda$.

Theorem 2. The value $V$ is decreasing in $\pi, \lambda$, and $\sigma$. The optimal liquidation level $B$ is decreasing in $\lambda$ and $\sigma$.

Proof. Recall that $\phi \mapsto F(\phi)$ is convex and satisfies $F(\phi)=1+\phi$ for $\phi \geq B$. It follows that

$$
(1+\phi) F^{\prime}(\phi)-F(\phi) \leq 0 .
$$

Using the relation $V=x F(\phi) /(1+\phi)$, we find that

$$
\frac{\partial V}{\partial \phi}=x \frac{\partial}{\partial \phi} \frac{F(\phi)}{1+\phi}=\frac{(1+\phi) F^{\prime}(\phi)-F(\phi)}{(1+\phi)^{2}} \leq 0 .
$$

Since $\pi=\phi /(1+\phi), \pi$ is increasing in $\phi$, so $V$ is also decreasing in $\pi$.

Next, let $\lambda_{2} \geq \lambda_{1}>0$ and $\sigma_{2} \geq \sigma_{1}>0$, and let $F_{1}$ and $F_{2}$ be the corresponding value functions defined by (7). Moreover, let $B_{1}$ and $B_{2}$ be the corresponding optimal liquidation levels, and let $\mathscr{L}_{1}$ and $\mathscr{L}_{2}$ be the corresponding linear operators defined by (6). For $y \leq B_{2}$, it follows from the fact that $\left(\mathcal{L}_{2}+\mu_{2}-\lambda_{2}-r\right) F_{2}=0$, the convexity of $F_{2}$, and (13) that

$$
\left(\mathcal{L}_{1}+\mu_{2}-\lambda_{1}-r\right) F_{2}=\frac{\phi^{2}\left(\mu_{2}-\mu_{1}\right)^{2}}{2}\left(\sigma_{1}^{-2}-\sigma_{2}^{-2}\right) F_{2}^{\prime \prime}+\left(\lambda_{1}-\lambda_{2}\right)\left((1+\phi) F_{2}^{\prime}-F_{2}\right) \geq 0 .
$$

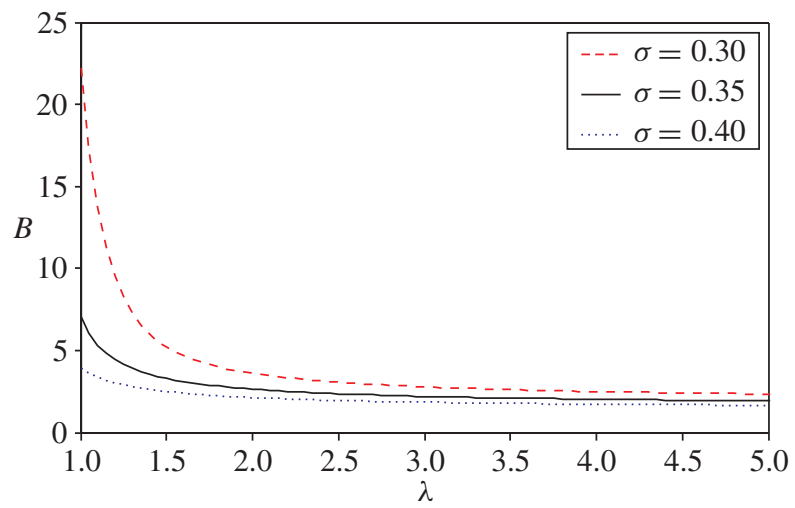

Figure 2: The optimal threshold $B$ as a function of the jump intensity $\lambda$ for three different values of the volatility $\sigma$. The model parameters are $\mu_{1}=-0.2, \mu_{2}=0.2$, and $r=0.05$. 
Thus, $F_{2}$ is superharmonic on $\left(0, B_{2}\right)$ with respect to the operator $\mathcal{L}_{1}+\mu_{2}-\lambda_{1}-r$, so if $\Phi$ is the process with parameters $\lambda_{1}$ and $\sigma_{1}$, then it is straightforward to check that

$$
F_{2}(\phi) \leq \mathbb{E}_{\phi} \mathrm{e}^{\left(\mu_{2}-\lambda_{1}-r\right) \tau_{B_{2}}} F_{2}\left(\Phi_{\tau_{B_{2}}}\right) .
$$

Consequently,

$$
F_{2}(\phi) \leq \mathbb{E}_{\phi} \mathrm{e}^{\left(\mu_{2}-\lambda_{1}-r\right) \tau_{B_{2}}} F_{2}\left(\Phi_{\tau_{B_{2}}}\right)=\mathbb{E}_{\phi} \mathrm{e}^{\left(\mu_{2}-\lambda_{1}-r\right) \tau_{B_{2}}}\left(1+\Phi_{\tau_{B_{2}}}\right) \leq F_{1}(\phi) .
$$

Note that if $\phi \geq B_{2}$ then $F_{2}(\phi)=1+\phi \leq F_{1}(\phi)$, so this inequality holds for all $\phi$. Consequently, $F$ (and, hence, also $V$ ) is decreasing in $\lambda$ and in $\sigma$. Since $B=\inf \{\phi: F(\phi)=$ $1+\phi$, it also follows that the optimal liquidation level $B$ is decreasing in $\lambda$ and in $\sigma$.

\section{Optimal closing of a short position}

In this section we consider the opposite situation in which the agent believes in a small initial drift, and that the small drift is replaced with a larger drift at a random and unobservable change point. To trade on this belief, the agent has a short position in the asset, and he/she seeks an optimal time to close the short position. As in the previous case, the optimal closing time would be precisely at the change point, but this is in general impossible due to the change point being unobservable.

Let $\theta$ be as in the previous sections, let $\mu_{1}<r<\mu_{2}$, and model $X$ by

$$
\mathrm{d} X_{t}=\mu(t) X_{t} \mathrm{~d} t+\sigma X_{t} \mathrm{~d} W_{t},
$$

where the drift now is given by

$$
\mu(t)=\mu_{1}+\left(\mu_{2}-\mu_{1}\right) \mathbf{1}_{\{t \geq \theta\}} .
$$

We define

$$
U=\inf _{\{\tau \in \mathcal{T}: \tau<\infty\}} \mathbb{E} \mathrm{e}^{-r \tau} X_{\tau} .
$$

Note that the infimum is taken over almost surely finite $\mathcal{F}^{X}$-stopping times (in this way we do not need to define the pay off for $\tau=\infty$ ). Define the a posteriori probability process $\Pi$ by

$$
\Pi_{t}=\mathbb{P}\left(\theta \leq t \mid \mathcal{F}_{t}^{X}\right),
$$

and let $\Phi_{t}=\Pi_{t} /\left(1-\Pi_{t}\right)$. By the same arguments as in the case of a long investor, we find that

$$
\mathbb{E}^{-r \tau} X_{\tau}=\frac{x}{1+\phi} \mathbb{E}^{\mathbb{Q}} \mathrm{e}^{\left(\mu_{2}-\lambda-r\right) \tau}\left(1+\Phi_{\tau}\right)
$$

for finite $\mathcal{F}^{X}$-stopping times $\tau$. Here $\Phi$ satisfies

$$
\mathrm{d} \Phi_{t}=\left(\lambda+(\lambda+\omega \sigma) \Phi_{t}\right) \mathrm{d} t+\omega \Phi_{t} \mathrm{~d} Z_{t},
$$

where $Z$ is a $\left\{\mathbb{Q}, \mathcal{F}^{X}\right\}$-Brownian motion. Consequently,

$$
U=\frac{x H(\phi)}{1+\phi}
$$

where

$$
H(\phi)=\inf _{\tau} \mathbb{E}_{\phi}^{Q} \mathrm{e}^{\left(\mu_{1}-\lambda-r\right) \tau}\left(1+\Phi_{\tau}\right)
$$


By Itô's formula, the drift of the process $\mathrm{e}^{\left(\mu_{1}-\lambda-r\right) t}\left(1+\Phi_{t}\right)$ is positive if $\Phi_{t}>$ $\left(r-\mu_{1}\right) /\left(\mu_{2}-r\right)$ and negative if $\Phi_{t}<\left(r-\mu_{1}\right) /\left(\mu_{2}-r\right)$. This suggests that the optimal stopping time is the first hitting time of the set $[A, \infty)$ for some level $A$. Moreover, the pair $(H, A)$ should satisfy the free boundary problem

$$
\begin{gathered}
\mathcal{L} H+\left(\mu_{1}-\lambda-r\right) H=0, \quad 0<\phi<A, \\
H(\phi)=1+\phi, \quad \phi \geq A ; \quad H^{\prime}(A)=1, \quad H(0+)<\infty,
\end{gathered}
$$

where

$$
\mathcal{L} H=\frac{\omega^{2} \phi^{2}}{2} H^{\prime \prime}+(\lambda+(\lambda+\omega \sigma) \phi) H^{\prime} .
$$

The differential equation in (16) has the general solution

$$
H(\phi)=C \psi(\phi)+D \varphi(\phi),
$$

where

$$
\psi(\phi)=\int_{0}^{\infty} \mathrm{e}^{-a t} t^{(b+\gamma-3) / 2}(1+\phi t)^{(\gamma-b+1) / 2} \mathrm{~d} t
$$

and

$$
\varphi(\phi)=\int_{0}^{1 / \phi} \mathrm{e}^{a t} t^{(b+\gamma-3) / 2}(1-\phi t)^{(\gamma-b+1) / 2} \mathrm{~d} t
$$

with

$$
\gamma=\sqrt{(b-1)^{2}+4 c}, \quad a=\frac{2 \lambda}{\omega^{2}}, \quad b=\frac{2}{\omega}\left(\frac{\lambda}{\omega}+\sigma\right), \quad \text { and } \quad c=\frac{2\left(\lambda+r-\mu_{1}\right)}{\omega^{2}} .
$$

Using the boundary conditions in (16), we arrive at the free boundary equation

$$
\begin{aligned}
2 \int_{0}^{\infty} & \mathrm{e}^{-a t} t^{(b+\gamma-3) / 2}(1+A t)^{(\gamma-b+1) / 2} \mathrm{~d} t \\
& =(\gamma-b+1)(1+A) \int_{0}^{\infty} \mathrm{e}^{-a t} t^{(b+\gamma-1) / 2}(1+A t)^{(\gamma-b-1) / 2} \mathrm{~d} t .
\end{aligned}
$$

The following result can then be proved along the same lines as Theorem 1 above.

Theorem 3. Let $A$ be the unique positive solution to the free boundary equation (17). Then

$$
H(\phi)= \begin{cases}\frac{1+A}{\psi(A)} \psi(\phi), & \phi<A \\ 1+\phi, & \phi \geq A\end{cases}
$$

Moreover, the stopping time $\tau_{A}:=\inf \left\{t \geq 0: \Phi_{t} \geq A\right\}$ is optimal in (15).

Furthermore, with $\varphi=\pi /(1-\pi)$ we have

$$
U= \begin{cases}\frac{(1+A) \psi(\varphi)}{(1+\varphi) \psi(A)} x, & \varphi<A, \\ x, & \varphi \geq A,\end{cases}
$$

and the supremum in (14) is attained for $\tau^{*}:=\inf \left\{t \geq 0: \Pi_{t} \geq A /(A+1)\right\}$. 


\section{Acknowledgements}

Support from the Swedish Research Council (Ekström) and the Gothenburg Mathematical Modelling Centre (Lindberg) is gratefully acknowledged.

\section{References}

[1] Beibel, M. And Lerche, H. R. (1997). A new look at optimal stopping problems related to mathematical finance. Statistica Sinica 7, 93-108.

[2] Blitz, D. C. ANd Vliet, P. (2008). Global tactical cross-asset allocation: applying value and momentum across asset classes. J. Portfolio Manag. 35, 23-38.

[3] Borodin, A. N. and Salminen, P. (2002). Handbook of Brownian Motion-Facts and Formulae, 2nd edn. Birkhäuser, Basel.

[4] Connolly, R. And Stivers, C. (2003). Momentum and reversals in equity-index returns during periods of abnormal turnover and return dispersion. J. Finance 58, 1521-1556.

[5] Cooper, M. J., Gutierrez, R. C., Jr. And Hameed, A. (2004). Market states and momentum. J. Finance 59, 1345-1365.

[6] Du Toit, J. And Peskir, G. (2009). Selling a stock at the ultimate maximum. Ann. Appl. Prob. 19, 983-1014.

[7] Eкström, E. AND Lu, B. (2011). Optimal selling of an asset under incomplete information. Internat. J. Stoch. Anal. 2011, 17pp.

[8] Figelman, I. (2007). Stock return momentum and reversal. J. Portfolio Manag. 34, 51-67.

[9] Gebhardt, W. R., Hvidkjaer, S. and Swaminathan, B. (2005). Stock and bond market interaction: does momentum spill over? J. Financial Econom. 75, 651-690.

[10] George T. J. And Hwang, C.-Y. (2004). The 52-week high and momentum investing. J. Finance 59, $2145-2176$.

[11] Graversen, S. E., Peskir, G. and Shiryaev, A. N. (2001). Stopping Brownian motion without anticipation as close as possible to its ultimate maximum. Theory Prob. Appl. 45, 41-50.

[12] Griffin, J. M., Ji, X. And Martin, J. S. (2003). Momentum investing and business cycle risk: evidence from pole to pole. J. Finance 58, 2515-2547.

[13] Grundy, B. D. And Martin, J. S. (2001). Understanding the nature of the risks and the source of the rewards to momentum investing. Rev. Financial Studies 14, 29-78.

[14] Gutierrez, R. C., Jr. And Kelley, E. K. (2008). The long-lasting momentum in weekly returns. J. Finance 63, 415-447.

[15] Hogan, S., Jarrow, R., Melvyn, T. and WarachKa, M. (2003). Testing market efficiency using statistical arbitrage with applications to momentum and value strategies. J. Financial Econom. 73, 525-565.

[16] Jegadeesh, N. and Titman, S. (1993). Returns to buying winners and selling losers: implications for stock market efficiency. J. Finance 48, 65-91.

[17] Jegadeesh, N. and Titman, S. (1995). Overreaction, delayed reaction, and contrarian profits. Rev. Financial Studies 8, 973-993.

[18] Jegadeesh, N. And Titman, S. (2001). Profitability of momentum strategies: an evaluation of alternative explanations. J. Finance 56, 699-720.

[19] Jegadeesh, N. and Titman, S. (2002). Cross-sectional and time-series determinants of momentum returns. Rev. Financial Studies 15, 143-157.

[20] Karatzas, I. And Shreve, S. E. (1998). Methods of Mathematical Finance (Appl. Math. 39). Springer, New York.

[21] Karatzas, I. And Shreve, S. E. (2000). Brownian Motion and Stochastic Calculus, 2nd edn. Springer, New York.

[22] KleIN, M. (2009). Comment on "Investment timing under incomplete information". Math. Operat. Res. 34, 249-254.

[23] KorajCZyK, R. A. AND SADKA, R. (2004). Are momentum profits robust to trading costs? J. Finance 59, 10391082.

[24] Lee, C. M. C. and Swaminathan, B. (2000). Price momentum and trading volume. J. Finance 55, 2017-2069.

[25] Lewellen, J. (2002). Momentum and autocorrelation in stock returns. Rev. Financial Studies 15, 533-564.

[26] Liptser, R. S. And Shiryaev, A. N. (1977). Statistics of Random Processes. I. Springer, New York.

[27] LiU, L. X. And Zhang, L. (2009). Momentum profits, factor pricing, and macroeconomic risk. Rev. Financial Studies 21, 2417-2448.

[28] Moskowitz, T. J. and Grinblatt, M. (1999). Do industries explain momentum? J. Finance 54, 1249-1290.

[29] ØKsendal, B. (2003). Stochastic Differential Equations, 6th edn. Springer, Berlin.

[30] Peskir, G. And Shiryaev, A. (2006). Optimal Stopping and Free-Boundary Problems. Birkhäuser, Basel .

[31] Rouwenhorst, K. G. (1998). International momentum strategies. J. Finance 53, 267-284.

[32] Shiryaev, A. N. (1978). Optimal Stopping Rules. Springer, New York. 
[33] Shiryaev, A. N. (2002). Quickest detection problems in the technical analysis of the financial data. In Mathematical Finance-Bachelier Congress (Paris, 2000), Springer, Berlin, pp. 487-521.

[34] Shiryaev, A. N. And Novikov, A. A. (2008). On a stochastic version of the trading rule "Buy and Hold". Statist. Decisions 26, 289-302.

[35] Shiryaev, A., Xu, Z. and Zhou, X. Y. (2008). Thou shalt buy and hold. Quant. Finance 8, 765-776.

[36] Stojanovic, S. (2004/05). Optimal momentum hedging via hypoelliptic reduced Mone-Ampère PDEs. SIAM J. Control Optimization 43, 1151-1173.

[37] Villeneuve, S. (2007). On threshold strategies and the smooth-fit principle for optimal stopping problems. J. Appl. Prob. 44, 181-198. 